\title{
Influence of Ultra-Low-Dose and Iterative Reconstructions on the Visualization of Orbital Soft Tissues on Maxillofacial CT
}

\author{
(D) G. Widmann, (DD. Juranek, DF. Waldenberger, (DP. Schullian, ${ }^{(D)}$ A. Dennhardt, ${ }^{D}$ R. Hoermann, ${ }^{(D)}$ M. Steurer, ${ }^{(D)}$ E.-M. Gassner, and \\ (D)W. Puelacher
}

\begin{abstract}
BACKGROUND AND PURPOSE: Dose reduction on CT scans for surgical planning and postoperative evaluation of midface and orbital fractures is an important concern. The purpose of this study was to evaluate the variability of various low-dose and iterative reconstruction techniques on the visualization of orbital soft tissues.

MATERIALS AND METHODS: Contrast-to-noise ratios of the optic nerve and inferior rectus muscle and subjective scores of a human cadaver were calculated from CT with a reference dose protocol (CT dose index volume $=36.69 \mathrm{mGy}$ ) and a subsequent series of low-dose protocols (LDPs I-4: CT dose index volume $=4.18,2.64,0.99$, and $0.53 \mathrm{mGy}$ ) with filtered back-projection (FBP) and adaptive statistical iterative reconstruction (ASIR)-50, ASIR-100, and model-based iterative reconstruction. The Dunn Multiple Comparison Test was used to compare each combination of protocols $(\alpha=.05)$.
\end{abstract}

RESULTS: Compared with the reference dose protocol with FBP, the following statistically significant differences in contrast-to-noise ratios were shown (all, $P \leq .012$ ) for the following: 1) optic nerve: LDP-I with FBP; LDP-II with FBP and ASIR-50; LDP-III with FBP, ASIR-50, and ASIR-100; and LDP-IV with FBP, ASIR-50, and ASIR-100; and 2) inferior rectus muscle: LDP-II with FBP, LDP-III with FBP and ASIR-50, and LDP-IV with FBP, ASIR-50, and ASIR-100. Model-based iterative reconstruction showed the best contrast-to-noise ratio in all images and provided similar subjective scores for LDP-II. ASIR-50 had no remarkable effect, and ASIR-100, a small effect on subjective scores.

CONCLUSIONS: Compared with a reference dose protocol with FBP, model-based iterative reconstruction may show similar diagnostic visibility of orbital soft tissues at a CT dose index volume of $2.64 \mathrm{mGy}$. Low-dose technology and iterative reconstruction technology may redefine current reference dose levels in maxillofacial CT.

ABBREVIATIONS: ALADA = As Low As Diagnostically Achievable; ALARA = As Low As Reasonably Achievable; ASIR = adaptive statistical iterative reconstruction; $\mathrm{CNR}=$ contrast-to-noise ratio; $\mathrm{CTDI}_{\text {vol }}=\mathrm{CT}$ dose index volume; $\mathrm{FBP}=$ filtered back-projection; $\mathrm{HU}=$ Hounsfield unit; $\mathrm{IRM}=$ inferior rectus muscle; $\mathrm{LDP}=$ low-dose protocol; MBIR = model-based iterative reconstruction; $\mathrm{ON}=$ optic nerve

$\mathrm{T}$ he success of modern maxillofacial surgery is undoubtedly related to the increased use of multisection CT. Image data are used for diagnosis and treatment, including software planning, fabrication of rapid prototyping models, customized surgical plate modeling, and computer-guided surgery.

However, the increasing use of CT has been cited as a cause for the increasing collective dose of ionizing radiation to populations. ${ }^{1}$ The

Received February 10, 2017; accepted March 19.

From the Departments of Radiology (G.W., P.S., M.S., E.-M.G.) and Craniomaxillofacial Surgery (D.J., F.W., A.D., W.P.) and Division of Functional and Clinical Anatomy (R.H.), Innsbruck Medical University, Innsbruck, Austria.

Paper previously presented in abstract form at: Computer-Assisted Radiology and Surgery 30th International Congress and Exhibition, June 21-25, 2016; Heidelberg, Germany.

Please address correspondence to Gerlig Widmann, MD, Department of Radiology, Innsbruck Medical University, A-6020 Innsbruck, Anichstr 35, Austria; e-mail: gerlig. widmann@i-med.ac.at

http://dx.doi.org/10.3174/ajnr.A5239 public awareness of cumulative radiation exposure from medical imaging is strongly reflected by legislative authorities and radiologic societies, leading to recent awareness campaigns such as the Image Gently and Image Wisely Campaigns and the American College of Radiology Dose Index Registry Initiatives. ${ }^{2}$

In the field of maxillofacial imaging, the eye lenses and thyroid gland are the most critical organs affected by direct or scattered radiation. ${ }^{3}$ Available CT technology has to be optimized to assure that the examination adheres to the As Low As Reasonably Achievable (ALARA)/As Low As Diagnostically Achievable (ALADA) principles to reduce the potential risks from ionizing radiation. Protection from ionizing radiation is critical in young and middle-aged patients who frequently have sports-related midface and orbital fractures. ${ }^{4}$ One has to take into account that most patients have already had an initial CT scan during the emergency diagnostic evaluation and may require repeat CT scans for treatment planning, guided surgery, and postoperative evaluation. ${ }^{5}$

Modern CT technology is able to substantially reduce the 
Table 1: Scan protocols and parameters used including CTDI ${ }_{\text {vol }}$, DLP, and effective dose

\begin{tabular}{|c|c|c|c|c|c|c|c|c|c|}
\hline Dose Protocol & $\begin{array}{l}\text { Reconstruction } \\
\text { Technique }\end{array}$ & Kernel & kV & mAs & Pitch & $\begin{array}{c}\text { Rotation } \\
\text { Time (s) }\end{array}$ & CTDI $_{\text {vol }}$ (mGy) & $\begin{array}{c}\text { DLP } \\
(\mathrm{mG} \times \mathrm{cm})\end{array}$ & $\begin{array}{c}\text { Effective } \\
\text { Dose (mSv) }\end{array}$ \\
\hline Emergency protocol head & ASIR-50 & Bone & 120 & $314-488$ & 1 & 0.6 & 47.55 & 1141.26 & 4.9 \\
\hline Reference dose protocol & $\begin{array}{l}\text { FBP } \\
\text { ASIR-50 } \\
\text { ASIR-100 } \\
\text { MBIR }\end{array}$ & $\begin{array}{l}\text { Bone, std } \\
\text { Bone, std } \\
\text { Bone, std } \\
\text { std }\end{array}$ & 120 & 100 & 0.5 & 1 & 36.69 & 930.78 & 3.5 \\
\hline Low-dose protocol I & $\begin{array}{l}\text { FBP } \\
\text { ASIR-50 } \\
\text { ASIR-100 } \\
\text { MBIR }\end{array}$ & $\begin{array}{l}\text { Bone, std } \\
\text { Bone, std } \\
\text { Bone, std } \\
\text { std }\end{array}$ & 100 & 35 & 0.5 & 0.5 & 4.18 & 106.18 & 0.4 \\
\hline Low-dose protocol II & $\begin{array}{l}\text { FBP } \\
\text { ASIR-50 } \\
\text { ASIR-100 } \\
\text { MBIR }\end{array}$ & $\begin{array}{l}\text { Bone, std } \\
\text { Bone, std } \\
\text { Bone, std } \\
\text { std }\end{array}$ & 80 & 40 & 0.5 & 0.5 & 2.64 & 66.69 & 0.3 \\
\hline Low-dose protocol III & $\begin{array}{l}\text { FBP } \\
\text { ASIR-50 } \\
\text { ASIR-100 } \\
\text { MBIR }\end{array}$ & $\begin{array}{l}\text { Bone, std } \\
\text { Bone, std } \\
\text { Bone, std } \\
\text { std }\end{array}$ & 80 & 15 & 0.5 & 0.5 & 0.99 & 25.13 & 0.1 \\
\hline Low-dose protocol IV & $\begin{array}{l}\text { FBP } \\
\text { ASIR-50 } \\
\text { ASIR-100 } \\
\text { MBIR }\end{array}$ & $\begin{array}{l}\text { Bone, std } \\
\text { Bone, std } \\
\text { Bone, std } \\
\text { std }\end{array}$ & 80 & 10 & 0.5 & 0.4 & 0.53 & 13.39 & 0.1 \\
\hline
\end{tabular}

Note:-DLP indicates dose-length product; std, standard.

dose. ${ }^{6-10}$ Furthermore, dose reduction may not significantly influence registration and navigation accuracy for computer-aided surgery. ${ }^{11-13}$ However, the associated increase in noise may significantly influence the diagnostic image quality of soft-tissue structures such as the optical nerve and orbital muscles. The recent implementation of iterative reconstruction techniques such as adaptive statistical iterative reconstruction (ASIR) and modelbased iterative reconstruction (MBIR) may improve image quality with reduced radiation doses compared with the traditionally used filtered back-projection (FBP) technique. ${ }^{14,15}$

The aim of the present study was to evaluate the influence of various low-dose protocols and iterative reconstructions on the visualization of orbital soft tissues.

\section{MATERIALS AND METHODS}

We used 1 cadaver head preserved with an arterial injection of a formaldehyde-phenol solution/an alcohol-glycerin solution and immersion in phenolic acid in water for $1-3$ months. ${ }^{16}$ The bodies were donated by individuals who had given their informed consent before death for their use for scientific and educational purposes. ${ }^{17,18}$

The entire cadaver head including the mandible was scanned by using the Discovery CT750 HD (GE Healthcare, Milwaukee, Wisconsin) and the following protocols: the standard emergency protocol of the head (as used during initial cranial CT in the emergency setting), a high-resolution reference dose protocol (as used for surgical planning and navigated surgery), and a subsequent series of lowdose protocols (LDPs) with various iterative reconstruction techniques and bone and standard kernels (protocol details can be found in Table 1). The images were exported in a DICOM format with Impax EE (Agfa-Gevaert, Mortsel, Belgium) PACS.

\section{Dose Estimation}

CT dose index volume $\left(\mathrm{CTDI}_{\mathrm{vol}}\right)$ and dose-length product were obtained from the DICOM tags. In addition, CT-Expol, Version 2.1 (Medical University Hannover, Hannover, Ger- many), an Excel (Microsoft, Redmond, Washington) application for assessing the radiation doses delivered to patients undergoing CT examinations based on the scan parameters of the used scanner type, was used to calculate effective doses (International Commission of Radiation Protection: ICRP 103; http://apps.snm.org/docs/CME/PresenterItems/EventID_ 85/PresenterItemTypeID_1/3.\%20Eckerman\%20-\%201050. pdf) (Table 1). ${ }^{19}$

\section{Analysis of Hounsfield Unit Values and Contrast-to-Noise Ratios of Orbital Soft Tissues}

All images were evaluated by 2 readers with AW Server 2.0 (GE Healthcare). The readers were trained for image reformation and volumetric segmentation.

Images were reformatted in axial, coronal, and sagittal views. The window/level was adjusted to obtain the best subjective contrast. The paint-on-slices tool of the AW Server 2.0 software was used to segment the optical nerve $(\mathrm{ON})$ and inferior rectus muscle (IRM) on both sides.

The anterior border of the segmented structures was the horizontal line at the posterior margin of the orbital bulb, and the posterior border was the horizontal line at the foramen of the posterior ethmoid artery. Average Hounsfield units (HUs) and SDs of the segmented volumes were recorded.

In addition, the average Hounsfield unit and SD of the orbital fat lateral to the optic nerve posterior to the horizontal line at the posterior margin of the orbital bulb was measured with a spheric ROI with a 6-mm diameter.

The contrast-to-noise ratio (CNR) for each anatomic structure (ON and IRM) was calculated with the following formula:

$$
C N R_{\mathrm{a}}=\frac{\left|M H U_{\mathrm{a}}-M H U_{\mathrm{fat}}\right|}{\sqrt{\left(S D_{\mathrm{a}}^{2}+S D_{\mathrm{fat}}^{2}\right)}},
$$

with $M H U$ indicating the mean Hounsfield unit value, and SD, the standard deviation of Hounsfield unit values. 

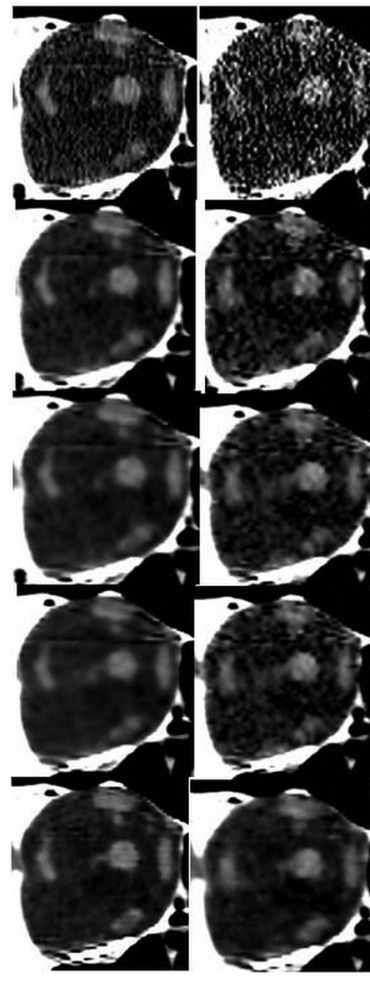

Low Dose II

Low Dose III Low Dose IV

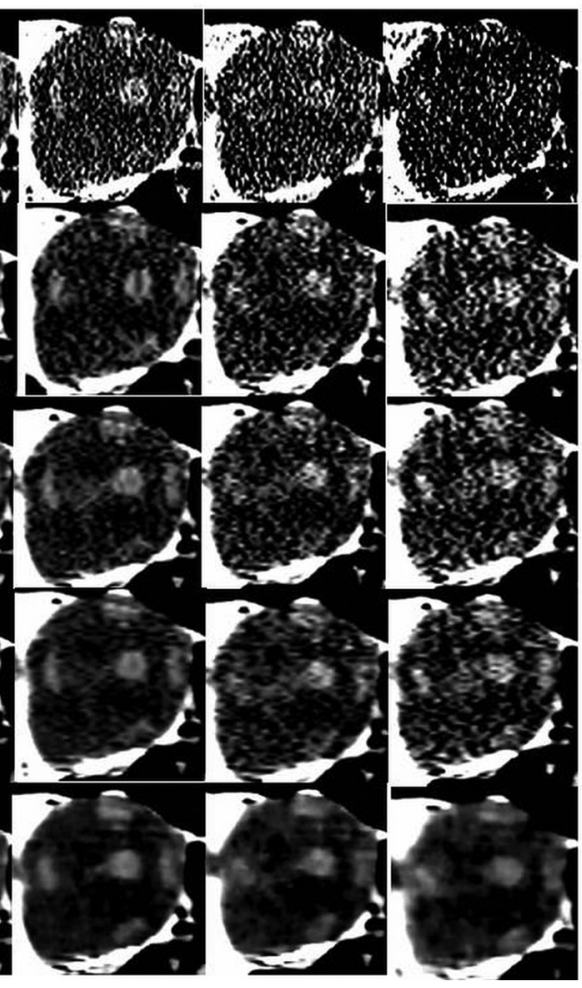

FIG 1. Images of the right orbit with different protocols (vertical columns), reconstructions, and kernels (horizontal columns). Std indicates standard.

Table 2: Mean CNR and SD of ON and IRM for each combination of protocol and reconstruction technique ${ }^{\mathrm{a}}$

\begin{tabular}{|c|c|c|c|c|c|}
\hline Dose Protocol & $\begin{array}{c}\text { Reconstruction } \\
\text { Technique }\end{array}$ & $\frac{\mathrm{ON}}{\mathrm{CNR}(\mathrm{SD})}$ & $\frac{\text { IRM }}{\text { CNR (SD) }}$ & $\begin{array}{c}\text { Score } \\
\text { Bone } \\
\text { Kernel }\end{array}$ & $\begin{array}{c}\text { Score } \\
\text { Std } \\
\text { Kernel }\end{array}$ \\
\hline Emergency protocol head & ASIR-50 & $2.75(0.62)$ & $1.82(0.26)$ & 4.0 & \\
\hline \multirow{4}{*}{ Reference dose protocol } & FBP & $3.80(2.07)$ & $2.39(1.22)$ & 3.0 & 4.0 \\
\hline & ASIR-50 & $4.28(2.04)$ & $2.71(1.24)$ & 3.0 & 4.0 \\
\hline & ASIR-100 & $4.37(1.39)$ & $2.99(1.01)$ & 3.0 & 4.0 \\
\hline & MBIR & $5.68(0.17)$ & $3.60(0.54)$ & & 5.0 \\
\hline \multirow[t]{4}{*}{ Low-dose protocol I } & FBP & $1.57(1.07)^{\mathrm{b}}$ & $1.11(0.79)$ & 2.0 & 3.0 \\
\hline & ASIR-50 & $2.15(1.27)$ & $1.22(0.81)$ & 2.0 & 3.0 \\
\hline & ASIR-100 & $2.49(0.98)$ & $1.65(0.59)$ & 2.0 & 3.0 \\
\hline & MBIR & $4.12(0.85)$ & $3.05(0.46)$ & & 4.0 \\
\hline \multirow[t]{4}{*}{ Low-dose protocol II } & FBP & $1.05(0.75)^{b}$ & $0.74(0.46)^{b}$ & 1.0 & 2.0 \\
\hline & ASIR-50 & $1.41(0.94)^{\mathrm{b}}$ & $0.91(0.43)$ & 1.0 & 2.0 \\
\hline & ASIR-100 & $2.16(1.15)$ & $1.34(0.40)$ & 1.0 & 3.0 \\
\hline & MBIR & $4.11(0.66)$ & $2.81(0.77)$ & & 4.0 \\
\hline \multirow[t]{4}{*}{ Low-dose protocol III } & FBP & $0.63(0.44)^{b}$ & $0.23(0.30)^{\mathrm{b}}$ & 1.0 & 1.0 \\
\hline & ASIR-50 & $0.87(0.57)^{\mathrm{b}}$ & $0.50\left(0.44^{b}\right.$ & 1.0 & 1.0 \\
\hline & ASIR-100 & $1.29(0.65)^{b}$ & $0.92(0.60)$ & 1.0 & 1.0 \\
\hline & MBIR & $3.08(0.63)$ & $2.32(0.44)$ & & 2.0 \\
\hline \multirow[t]{4}{*}{ Low-dose protocol IV } & FBP & $0.44(0.36)^{b}$ & $0.10(0.16)^{b}$ & 1.0 & 1.0 \\
\hline & ASIR-50 & $0.52(0.57)^{b}$ & $0.31(0.34)^{\mathrm{b}}$ & 1.0 & 1.0 \\
\hline & ASIR-100 & $0.93(0.63)^{b}$ & $0.62(0.56)^{b}$ & 1.0 & 1.0 \\
\hline & MBIR & $2.62(0.52)$ & $3.14(2.71)$ & & 2.0 \\
\hline
\end{tabular}

Note:-Std indicates standard.

a Subjective scores are given separately for images with a bone and standard kernel: 1 indicates very poor/not sufficient;

2 , suboptimal but sufficient; 3 , average = equal to reference; 4 , above average; and 5 , excellent.

${ }^{b}$ Statistically significant difference compared with the reference dose protocol with $\operatorname{FBP}(P<.05)$.

\section{Analysis of Subjective Image Quality}

Subjective image quality of the orbital soft tissues was assessed by consensus of 3 readers with a 5 -scale system: 1 , very poor/not sufficient; 2 , suboptimal but sufficient; 3 , average $=$ equal to ref-
FBP bone

FBP std

ASIR 50 std

ASIR 100 std

erence; 4, above average; and 5, excellent. As a "reference," the image of the reference dose protocol with FBP and the bone kernel was used, which referred to images usually provided by maxillofacial and sinus CT. Subjective scores were evaluated for each combination of dose, reconstruction technique, and kernel.

\section{Statistical Analysis}

Data analysis and descriptive statistics were performed with PASW Statistics (Version 15.0; IBM, Armonk, New York). Interobserver agreement was assessed with weighted $\kappa$ statistics. The Dunn Multiple Comparison Test and the Bonferroni-corrected test were used to compare each combination of protocols $(\alpha=.05)$. The analysis of variance for dependent variables was used to test between-subject effects: protocol, reconstruction, and kernel.

\section{RESULTS}

Using the 6 protocols, 4 reconstruction techniques, and 2 convolution kernels, we generated $36 \mathrm{CT}$ datasets. $\mathrm{CTDI}_{\mathrm{vol}}$, dose-length product, and effective doses can be found in Table 1 .

Compared with the reference dose protocol, LDP I-IV showed dose reductions of about $88.7 \%, 92.8 \%, 97.3 \%$, and 98.6\%. The effective doses for LDP I-IV, which include the scan of the entire head and mandible, were $0.4,0.3,0.1$, and 0.1 $\mathrm{mSv}$.

Figure 1 shows images including the reference dose protocol with FBP using the bone and standard kernels, and all low-dose protocols with FBP, ASIR-50, ASIR-100, and MBIR with the standard kernel. The mean CNR and SDs of the ON and IRM for each protocol and reconstruction technique can be found in Table 2 .

\section{Influence of Dose}

With the bone kernel, the reference dose protocol showed statistically significantly better CNR than all LDPs for both the ON and IRM (all $P<.001$ ). Compared with the reference standard, no statistically significant difference from the emergency protocol head $(P=1.000$ for the ON, $P=.951$ for IRM) was found. LDP-I was statistically significantly better than LDP II-IV for the ON $(P=.011, P<$ $.001, P<.001$ ), and LDP-III and IV, for the IRM (all, $P<.001$ ). 
LDP-I was not statistically significantly better than LDP-II for the $\operatorname{IRM}(P=1.000)$. LDP-II was statistically significantly better than LDP-III and IV for the ON $(P=.010, P<.001)$ and IRM (all $P<$ $.001)$. LDP-III did not show statistically significant differences from LDP-IV for the ON $(P=.112)$ and IRM $(P=.126)$.

With the standard kernel, the reference dose protocol showed statistically significantly better CNR than all LDPs (all $P<.001$ ). LDP-I was statistically significantly better than LDP III-IV for the ON (all, $P<.001$ ) and IRM (all $P=.004$ ). LDP-I was not statistically significantly better than LDP-II for the ON $(P=$ $.057)$ and IRM $(P=.611)$. LDP-II was statistically significantly better than LDP III and IV for the ON (all, $P<.001$ ) but not for the IRM $(P=.676, P=.759)$. LDP-III did not show statistically significant differences compared with LDP-IV for the ON $(P=.565)$ and IRM $(P=1.000)$.

\section{Influence of Reconstruction}

With the bone kernel, ASIR-50 and ASIR-100 showed statistically significantly better CNR than FBP for the ON and IRM (all $P<$ .001). ASIR-100 had a statistically significantly better CNR than ASIR-50 for the ON $(P=.007)$ and IRM $(P<.001)$.

With the standard kernel, ASIR-50 had a statistically significantly better CNR than FBP for the ON $(P=.037)$ but not for the $\operatorname{IRM}(P=1.000)$. ASIR-100 and MBIR showed statistically significantly better CNR than FBP for the ON (all, $P<.001$ ) and IRM $(P=.036, P<.001)$. ASIR-100 did not show a statistically significantly better CNR than ASIR-50 $(P=.745)$. MBIR showed a statistically significantly better CNR than both ASIR-50 and ASIR-100 for the ON (all $P<.001)$ and IRM $(P<.001, P=.001)$.

\section{Influence of the Convolution Kernel}

The standard kernel showed a statistically significantly higher CNR than the bone kernel for the ON and IRM (all $P<.001$ ). The convolution kernel influenced the effect of iterative reconstructions (see previous sections).

\section{Interobserver Agreement}

Interobserver agreement between both readers showed a Pearson correlation coefficient of 0.638 for the CNR measurements of the $\mathrm{ON}$ and 0.914 for the CNR measurements of the IRM. The Test of Between-Subjects Effects showed no statistically significant difference $(P=.950)$.

\section{Comparison with the Reference Dose Protocol with FBP}

Compared with the reference dose protocol with FBP, statistically significant differences of the CNR for the ON were shown with LDP-I with FBP $(P=.010)$; LDP-II with FBP $(P<.001)$ and ASIR-50 $(P=.003)$; LDP-III with FBP $(P<.001)$, ASIR-50 $(P<.001)$, and ASIR-100 $(P=.001)$; and LDP-IV with FBP $(P<.001)$, ASIR-50 $(P<.001)$, and ASIR-100 $(P<$ .001) (Table 2).

For the IRM, statistically significant differences from the reference dose protocol with FBP were shown with LDP-II with FBP $(P=.012)$; LDP-III with FBP $(P<.001)$ and ASIR-50 $(P=.001)$; and LDP-IV with FBP $(P<.001)$, ASIR-50 $(P<.001)$, and ASIR$100(P=.004)$ (Table 2).

\section{Subjective Scoring}

Compared with the reference dose protocol with FBP bone, which was given the reference score of 3 (average $=$ equal to reference), scores decreased progressively during dose reduction (see Table 2 and the comparison of the images in Fig 1). Images with a standard kernel showed a higher score than with the bone kernel. The reference dose protocol with FBP standard showed a score of 4 . In LDP-II, the standard kernel could retain a score of 2 (suboptimal but sufficient) in contrast to the bone kernel, which was insufficient. ASIR-50 had no remarkable effect over FBP on subjective scores. ASIR-100 showed a small effect. MBIR showed the best subjective score in all images. MBIR obtained a score of 4 for LDP I and II and retained a score of 2 for LDP III and IV. ON showed better subjective visibility then IRM.

\section{DISCUSSION}

Various iterative reconstruction technologies have been implemented to reduce image noise and improve the quality of lowdose images. ${ }^{20,21}$ Adaptive statistical iterative reconstruction uses information obtained from the FBP algorithm but integrates a comparison of the pixel values with an ideal value to selectively identify and then subtract noise from an image at adaptive blend levels freely selectable typically from $10 \%$ to $100 \% .^{22}$ Modelbased iterative reconstruction does not rely on the FBP as a starting point but instead uses a more complex system of prediction models, including noise and the spatial and geometric features of the $\mathrm{x}$-ray beam and detector technology. ${ }^{23}$ In a cadaver, ASIR-100 at a CTDI ${ }_{\mathrm{vol}}=3.48$ and $2.19 \mathrm{mGy}$ and MBIR at a CTDI $\mathrm{vol}_{\mathrm{vol}}=0.82$ $\mathrm{mGy}$ provided similar subjective cross-sectional and $3 \mathrm{D}$ bone image quality compared with the FBP reference at $\mathrm{CTDI}_{\mathrm{vol}}=30.48$ mGy. ${ }^{24}$ At a CTDI ${ }_{\mathrm{vol}}=2.19 \mathrm{mGy}$, ASIR-50 reduced noise by $22 \%$; ASIR-100, by $35 \%$; and MBIR, by $67 \% .{ }^{24}$ When we compared contrast-to-noise ratios of the different reconstructions using a reference dose protocol at a $\mathrm{CTDI}_{\mathrm{vol}}=36.58 \mathrm{mGy}$ and several ultra-low doses in a phantom study with a customized polymethyl methacrylate phantom, MBIR demonstrated the highest CNR throughout, followed by ASIR-100, ASIR-50, and FBP. ${ }^{25}$ With MBIR, ultra-low-dose protocols at CTDI $_{\mathrm{vol}}=4.14$ and $2.63 \mathrm{mGy}$ may show comparable scores for spatial resolution, which may allow a dose-reduction potential of up to $93 \% .{ }^{26}$ Compared with FBP, ASIR was not able to significantly improve spatial resolution. ${ }^{26}$ In a more recent human cadaver study evaluating the influence of various low-dose protocols and IRTs on the detectability of midfacial fractures, ultra-low-dose protocols at CTDI $_{\mathrm{vol}}=$ 1.0 and $2.6 \mathrm{mGy}$ were diagnostic for dislocated and nondislocated fractures, respectively. ${ }^{14}$ However, due to smoothing effects, ASIR and MBIR could not improve fracture detection. ${ }^{14}$ MBIR does not evaluate thin bony structures well; this evaluation may be important in surgical planning and postoperative evaluation. Unfortunately, MBIR cannot be reconstructed in bone kernels. ${ }^{14,27}$

Following the fracture study, the aim of the present study was to evaluate the variability of the CNR of orbital soft tissues with low-dose protocols and IRTs. The measures were based on $3 \mathrm{D}$ volume-segmented HU evaluations of the ON, IRM, and orbital fat. Compared with the reference dose protocol $\left(\mathrm{CTDI}_{\mathrm{vol}}=36.69\right.$ $\mathrm{mGy})$, LDP I-IV $\left(\mathrm{CTDI}_{\mathrm{vol}}=4.18,2.64,0.99\right.$, and $\left.0.53 \mathrm{mGy}\right)$ showed a dose reduction of up to $98.6 \%$. For comparison, the 
current reference dose of the German Federal Office for Radiation Protection for diagnostic sinus CT is $\mathrm{CTDI}_{\mathrm{vol}}=9 \mathrm{mGy}$. The presented doses described above were equal or lower than those of previous CT techniques and also most dentomaxillofacial conebeam CT devices. ${ }^{8-10,28,29}$ Unlike CT, an important limitation of conebeam CT is the insufficient soft-tissue contrast from which orbital soft tissues cannot be discriminated.

As expected, the reference dose protocol showed a statistically significantly better CNR than all LDPs for both the ON and IRM. The standard kernel had a statistically significantly higher CNR than the bone kernel, and the convolution kernel influenced the effect of iterative reconstructions. LDP-I showed a statistically significantly higher CNR than LDP-III and IV with both kernels. However, with a standard kernel, the CNR of LDP-I did not significantly differ from that of LDP-II. ASIR-50 showed significantly better CNR than FBP for both the ON and IRM with the bone kernel but only for the ON with the standard kernel. ASIR100 was statistically significantly better than ASIR-50 with the bone kernel but not if the standard kernel was used. Schulz et $\mathrm{al}^{21}$ reported that iterative reconstructions with a soft-tissue kernel were more efficient at $120 \mathrm{kV}$ than the $100-\mathrm{kV}$ series, and at the lowest dose settings, use of the soft-tissue kernel reduced the benefit of iterative reconstructions. In the present study, MBIR achieved a statistically significantly better CNR compared with all other reconstruction techniques, which is in line with previously published results with a phantom. ${ }^{25}$ Hoxworth et $\mathrm{al}^{27}$ reported a significant improvement in the evaluation of soft-tissue structures with MBIR. Compared with the reference dose protocol with FBP, CNR of both the ON and IRM showed no statistically significant difference from the following: 1) LDP-I with ASIR-50, ASIR-100, and MBIR; 2) LDP-II with ASIR-100 and MBIR; 3) LDP-III with MBIR; and 4) LDP-IV with MBIR.

The CNR change was also supported by the subjective scoring. Scores were lower with a decreasing dose and higher for the standard kernel than for the bone kernel. The ON showed better subjective visibility then the IRM. On maxillofacial CT, images are usually provided in only the bone kernel. With the bone kernel, the orbital soft tissues were highly visible with the reference dose. However, they were blurred and invisible with ultra-low doses. The standard kernel, which is typically used only for soft-tissue imaging, such as in oncology, significantly improved visibility. ASIR-50 had no remarkable effect over FBP on subjective scores, and ASIR-100 showed only a small effect. In agreement with other researchers, an insufficient number of photons may not be compensated for by simply increasing the iterative reconstruction strength. ${ }^{21}$ MBIR showed the best subjective score in all images. Compared with the reference dose protocol with FBP bone, which was given a score of 3 (average $=$ equal to reference) and the reference dose protocol with FBP standard, which obtained a score of 4 (above average), use of MBIR obtained a score of 4 for LDP-I and II and was able to retain a score of 2 (suboptimal but sufficient) for LDP-III and IV. Previous studies also indicated the high subjective image quality with iterative reconstructions in low-dose protocols, which may reach equivalent or even superior levels compared with non-dose-reduced CT scans with conventional FBP. ${ }^{15,24}$

The study has several limitations. It was performed with a human cadaver. The results may thus not be comparable in living humans. Only 1 cadaver head was used; however, the relative differences in CNR may be very similar. Intraorbital bleeding may impair the CNR of orbital soft tissues. The influence of artifacts from surgical screws and plates has not been investigated.

\section{CONCLUSIONS}

Application of optimally tailored CT protocols with the lowest acceptable diagnostic dose is imperative in modern radiology. High-resolution ultra-low-dose CT protocols capable of being used for surgical planning and image-guided maxillofacial surgery may retain similar diagnostic visibility of orbital soft tissues at $\mathrm{CTDI}_{\mathrm{vol}}=2.64 \mathrm{mGy}$ with MBIR compared with a reference protocol at $\mathrm{CTDI}_{\mathrm{vol}}=36.69 \mathrm{mGy}$ with FBP. Reconstructions with a standard kernel may improve orbital soft-tissue visibility and should be provided in addition to the bone kernel. The achieved diagnostic dose levels in modern CT may be far lower than current reference levels for sinus CT. Application of low-dose technology and iterative reconstructions should be tested in clinical practice and may lead to redefinition of the ALARA/ALADA criteria for maxillofacial CT.

\section{ACKNOWLEDGMENTS}

The authors wish to thank individuals who donated their bodies and tissues for the advancement of education and research.

Disclosures: Wolfgang Puelacher-UNRELATED: Employment: University Clinic for Craniomaxillofacial Surgery, Innsbruck, Austria.

\section{REFERENCES}

1. United Nations Scientific Committee on the Effects of Atomic Radiation. Sources and effects of ionizing radiation. Official Records of the General Assembly, Sixty-third Session, Supplement No. 46. New York: United Nations; 2008 http://www.unscear.org/docs/reports/ 2008/09-86753_Report_2008_GA_Report_corr2.pdf. Accessed May 1, 2017

2. Kalra MK, Sodickson AD, Mayo-Smith WW. CT radiation: key concepts for gentle and wise use. Radiographics 2015;35:1706-21 CrossRef Medline

3. Stewart FA, Akleyev AV, Hauer-Jensen M, et al; Authors on behalf of ICRP. ICRP publication 118: ICRP statement on tissue reactions and early and late effects of radiation in normal tissues and organs: threshold doses for tissue reactions in a radiation protection context. Ann ICRP 2012;41:1-322 CrossRef Medline

4. Kraft A, Abermann E, Stigler R, et al. Craniomaxillofacial trauma: synopsis of 14,654 cases with 35,129 injuries in 15 years. Craniomaxillofac Trauma Reconstr 2012;5:41-50 CrossRef Medline

5. Wick MC, Weiss RJ, Lill M, et al. The "Innsbruck Emergency Algorithm" avoids the underdiagnosis of blunt cervical vascular injuries. Arch Orthop Trauma Surg 2010;130:1269-74 CrossRef Medline

6. Jeong DK, Lee SC, Huh $\mathrm{KH}$, et al. Comparison of effective dose for imaging of mandible between multi-detector CT and cone-beam CT. Imaging Sci Dent 2012;42:65-70 CrossRef Medline

7. Cordasco G, Portelli M, Militi A, et al. Low-dose protocol of the spiral CT in orthodontics: comparative evaluation of entrance skin dose with traditional x-ray techniques. Prog Orthod 2013;14:24 CrossRef Medline

8. Bodelle B, Wichmann JL, Klotz N, et al. Seventy kilovolt ultra-low dose CT of the paranasal sinus: first clinical results. Clin Radiol 2015;70:711-15 CrossRef Medline

9. Lell MM, May MS, Brand M, et al. Imaging the parasinus region with a third-generation dual-source $\mathrm{CT}$ and the effect of tin filtration on 
image quality and radiation dose. AJNR Am J Neuroradiol 2015;36: 1225-30 CrossRef Medline

10. Schell B, Bauer RW, Lehnert T, et al. Low-dose computed tomography of the paranasal sinus and facial skull using a high-pitch dualsource system: first clinical results. Eur Radiol 2011;21:107-12 CrossRef Medline

11. Widmann G, Fasser M, Schullian P, et al. Substantial dose reduction in modern multi-slice spiral computed tomography (MSCT)guided craniofacial and skull base surgery. Rofo 2012;184:136-42 CrossRef Medline

12. Dubach P, Eichenberger A, Caversaccio M. Radiation dose reduction in computer assisted navigation for functional endoscopic sinus surgery: cadaver head experiments and clinical implementation. Rhinology 2010;48:339-43 CrossRef Medline

13. Nauer CB, Eichenberger A, Dubach P, et al. CT radiation dose for computer-assisted endoscopic sinus surgery: dose survey and determination of dose-reduction limits. AJNR Am J Neuroradiol 2009; 30:617-22 CrossRef Medline

14. Widmann G, Dalla Torre D, Hoermann R, et al. Ultralow-dose computed tomography imaging for surgery of midfacial and orbital fractures using ASIR and MBIR. Int J Oral Maxillofac Surg 2015;44: 441-46 CrossRef Medline

15. Bulla S, Blanke P, Hassepass F, et al. Reducing the radiation dose for low-dose CT of the paranasal sinuses using iterative reconstruction: feasibility and image quality. Eur J Radiol 2012;81:2246-50 CrossRef Medline

16. Platzer W, Putz R, Poisel S. New system for the preservation and storage of anatomical matter [in German]. Acta Anat (Basel) 1978; 102:60-67 CrossRef Medline

17. McHanwell S, Brenner E, Chirculescu AR, et al. The legal and ethical framework governing body donation in Europe: a review of current practice and recommendations for good practice. Eur JAnat 2008;12:12

18. Riederer BM, Bolt S, Brenner E, et al. The legal and ethical framework governing body donation in Europe: 1 st update on current practice. Eur J Anat 2012;16:21

19. Stamm G, Nagel HD. CT-expo: a novel program for dose evaluation in CT [in German]. Rofo 2002;174:1570-76 CrossRef Medline
20. Padole A, Ali Khawaja RD, Kalra MK, et al. CT radiation dose and iterative reconstruction techniques. AJR Am J Roentgenol 2015;204: W384-92 CrossRef Medline

21. Schulz B, Beeres M, Bodelle B, et al. Performance of iterative image reconstruction in $\mathrm{CT}$ of the paranasal sinuses: a phantom study. AJNR Am J Neuroradiol 2013;34:1072-76 CrossRef Medline

22. Silva AC, Lawder HJ, Hara A, et al. Innovations in CT dose reduction strategy: application of the adaptive statistical iterative reconstruction algorithm. AJR Am J Roentgenol 2010;194:191-99 CrossRef Medline

23. Fleischmann D, Boas FE. Computed tomography: old ideas and new technology. Eur Radiol 2011;21:510-17 CrossRef Medline

24. Widmann G, Schullian P, Gassner EM, et al. Ultralow-dose CT of the craniofacial bone for navigated surgery using adaptive statistical iterative reconstruction and model-based iterative reconstruction: 2D and 3D image quality. AJR Am J Roentgenol 2015;204:563-69 CrossRef Medline

25. Widmann G, Bischel A, Stratis A, et al. Ultralow dose dentomaxillofacial CT imaging and iterative reconstruction techniques: variability of Hounsfield units and contrast-to-noise ratio. Br J Radiol 2016; 89:20151055 CrossRef Medline

26. Widmann G, Bischel A, Stratis A, et al. Spatial and contrast resolution of ultralow dose dentomaxillofacial CT imaging using iterative reconstruction technology. Dentomaxillofac Radiol 2017 Feb 17. [Epub ahead of print] CrossRef Medline

27. Hoxworth JM, Lal D, Fletcher GP, et al. Radiation dose reduction in paranasal sinus CT using model-based iterative reconstruction. AJNR Am J Neuroradiol 2014;35:644-49 CrossRef Medline

28. Pauwels R, Beinsberger J, Collaert B, et al; SEDENTEXCT Project Consortium. Effective dose range for dental cone beam computed tomography scanners. Eur J Radiol 2012;81:267-71 CrossRef Medline

29. Bornstein MM, Scarfe WC, Vaughn VM, et al. Cone beam computed tomography in implant dentistry: a systematic review focusing on guidelines, indications, and radiation dose risks. Int J Oral Maxillofac Implants 2014;29(suppl):55-77 CrossRef Medline 